\section{Compliance improvement of compression therapy in patients with lymphedema}

\author{
Franz-Josef Schingale \\ Lympho-Opt Clinik, Pommelsbrunn, \\ Germany
}

\begin{abstract}
Lymphoedema is a progressive, chronic disease as a result of primary or secondary damage to the Lymph system consecutive increase and change in the interstitial tissue fluid, characterized by an alteration of tissues, with an increase of connective tissue and fat, as well as changes in extracellular matrix (hyaluronic acid, collagen, characterized glycosaminoglycans).

Compression therapy is recognized as an effective treatment in the management of venous and lymphatic disease since thousands of years.

Compression decreases filtration rate, reduces the venous reflux, improves the venous pumping function, reduces the ambulatory venous hypertension, increases the arterio-venous pressure gradient and improves the lymphatic drainage.
\end{abstract}

\section{What kind of compression do we need?}

Inelastic for the phase 1, the phase of reduction and elastic for phase 2 , the phase for maintaining the result.

The first problem is in phase 1: Inelastic bandages will slip down after about 5 hours due to reduction of the oedema.

Ideal goal of therapy is to normalize the lymphatic transport. Because of the chronic nature of lymphedema the therapeutic goal is to return the disease in the latent stage (limited transport capacity without lymphedema) or at least in the stage I and thereby achieve sustainable relief from the discomfort. In treatment planning one or more of the following sub-objectives have to be specified: i) improve lymphatic drainage; ii) softening fibrosclerotic tissue changes; iii) reduction of connective tissue; iv) improving the function deficits of the limbs, to enhance the effectiveness of muscle and joint pump; v) mediation of self-treatment options (skin care, certain lymph drainage handles, technique of lymphatic compression bandage); vi) repatriation or reintegration of such persons in their social environment such as school, training, study or job; vii) prevention of long-term care; viii) improving quality of life.

The most important column of the treatment is compression therapy and movement. We educate our patients in bandaging, but it is problematic, because the bandage slips after 5 hours and it was a disaster for the patient to take off the whole bandage and start again.

\section{Velcro devices}

That was the reason why we changed to JuxtaFit for self-management, a stiff bandage with velcro closures and a BPS Guide.

\section{Advantages for the patient}

It takes less time and the patient can readjust it after a few hours when the normal bandage starts to slip to prevent slipage and control the necessary compression. Further there is a better reduction due to higher stiffness.

In phase 2 of treatment, the oedema is reduced and for conservation of the result we need MTM hosiery.

\section{Stockings}

There are two different kinds of hosiery: round- and flat-knitted. Roundknitted is only in a few cases of lymphoedema possible. In most cases we need flat-knitted garment, this simulates the compression bandage by higher stiffness and less elasticity.

The garment of the elastic compression therapy has to be similar to the bandages, must be a hosiery that fits perfect, that is as effective as possible and is suitable for every day life and is particulary important for lymphedema.

To improve the compliance, the stocking companies use high quality compression garments with low elasticity which are easy for donning and doffing and have controlled therapeutic pressure, with anti-odour and anti-microbial fabrics, latex free for skin friendly wearing comfort. In the meantime there are different colours and patterns available.

Adaptive, high-quality compression care for the treatment of lymphatic lymphatic disorders makes it possible. It makes it possible to integrate the patient in the treatment of lymph disorders at an early stage and improves compliance in connection with compression treatment.
Correspondence: Lympho-Opt Clinik, Happurgerstr. 15, 91224 Pommelsbrunn, Germany.

Tel.:+49.9154.911.200

E-mail: schingale@lympho-opt.de

This work is licensed under a Creative Commons Attribution 4.0 License (by-nc 4.0).

(C) Copyright F-J. Schingale, 2018

Licensee PAGEPress, Italy

Veins and Lymphatics 2018; 7:7635

doi:10.4081/vl.2018.7635

\section{Mesurement}

The measuring points for lymphological garments are basically the same as those for phlebological hosiery.

If there is a deep skin fold at the measuring point, the point is moved.

Differences to round-knitted garments are due to: different stocking material (flatknitted), it can give the limb a better shape.

Compression that must act more deeply depending from the localisation of the oedema, fibrosis in lymphoedema. This can be done by oblique endings and integration of zippers, pockets, paddings etc.

In lymph and lipoedema the measurements generally have to be taken under tension (for flat-knitted compression stockings only). This method is used in order to achieve a similar effect as short-stretch bandages.

The robust, strong material is slightly prestretched, so that with the remaining slight elasticity can keep the oedema under control.

The intensity of the tension must be individually adapted to the patient's tissues, the skin quality, age and physical fitness (mobility, strength and so on).

Preparation by the therapist should be taken into account as well as the strain due to long periods of standing or sitting at work.

The harder the tissue and the longer the lymphoedema has existed without treatment, the more pressure is required.

As compression is the most important column of treatment of lymphatic disorders, we should be able to control the compliance.

Right now we have to believe what the patient will tell us about wearing his hosiery.

In the future we should be able to control the time of wearing garments by a chip.

Nowadays we could ask the patient if he is willing to be controlled by signing a document with his agreement. 